\title{
The impact of mechanic force on proliferative signaling molecules during liver regeneration
}

\begin{abstract}
Liver, as the central regulator of metabolism, plays numerous functions in human body. When account acute injuries, the liver processes the specific competence to regenerate and return to a constant size. Many signaling molecules have been discovered for the initiation of liver regeneration. However, few mechanical changes have been addressed in the past in terms of liver regeneration and size control. This review discussed the mechanisms of mechanosensing molecules Yap/TAZ, 14-3-3 and their functions in liver regeneration, and how hepatocytes swelling, a physical change after acute liver injury, may initiate liver regeneration.
\end{abstract}

Keywords: liver regeneration yap/taz, hepatocyte, swelling, mechanosensing, mechanotransduction 14-3-3
Volume I Issue 2 - 2015

\author{
Qiongqiong Zhou,' Cheng Zhang ${ }^{2}$ \\ 'Biomedical Sciences Department, Missouri State University, \\ USA \\ 2Division of Gastroenterology, Hepatology \& Nutrition, Ohio \\ State University, USA
}

Correspondence: Cheng Zhang, Department of Medicine, Division of Gastroenterology, Hepatology, \& Nutrition. The Ohio State University, Columbus, $\mathrm{OH} 43210$, the 2nd Floor, 395 W I2th Ave, USA, Tel (6I4) 2936255,

Email Cheng.Zhang@osumc.edu

Received:June 19,2015 | Published: August 17,2015
Abbreviations: YAP, yes-associated protein; TAZ, tafazzin; TEAD, tea domain family member; GPCR, g protein coupled receptor

\section{Introduction}

Liver, as the central regulator of metabolism, plays numerous functions in human body, including regulation of glycogen storage, decomposition of red blood cells, plasma protein synthesis, hormone production, and detoxification. A variety of facts can cause the liver malfunction and even lead to liver injury certain drugs like excessive amounts of acetaminophen, alcohol abuse, hepatitis viruses, cirrhosis, fatty liver disease, trauma, and so on. When the injury is acute and in a short period of time, the liver processes the specific competence to return to a constant size..$^{1-3}$ This regeneration capability can be observed clinically after acute liver injury, as well as experimentally in animal models after induced acute liver injury. Understanding this process has great benefits in the treatment of liver injury and fascinated biologist for many decades. Although there has been great advancement in understanding of the regenerative mechanism, the molecular signals responsible for mediating the injury to cell proliferation are not very clear; neither does the signals for maintaining an original liver volume. The aim of this review is to summarize recent progress in understanding liver regeneration and introduce the idea of mechanosensing in organ size maintenance.

In responding to acute liver injury, the hepatocytes are the main cell type to proliferate and thus to restore the mass of liver. However when injury is severe or when hepatocytes have lost the proliferative capacity, the reserved progenitor cells also called "oval" cells, are activated for proliferation. ${ }^{4}$ The proliferation of hepatocytes upon liver injury is proposed to be initiated by cytokine cascade in Kupffer cells, whereas in the activation of progenitor cells, multiple autocrine and paracrine factors have been reported in recent publications. ${ }^{5,6}$ However, the importance of how mechanic changes may trigger the proliferation of hepatocytes and progenitor cells have not been clearly addressed.

\section{Signaling molecules involving mechanosensing in liver regeneration:Yap/TAZ}

The Hippo signaling pathway is an evolutionarily conserved signaling module that plays critical roles in organ size control including liver. Yap and TAZ are key players in Hippo pathway when they are non-phosphorylated, Yap/TAZ localize to the nuclear and promote cell proliferation by regulating the activity of different transcription factors, including the TEA-domain-containing proteins (TEADs); when Yap/ TAZ are phosphorylated, they bind to $14-3-3$, resulting cytoplasmic retention and loss of transcriptional activity, thus cell proliferation is inhibited. ${ }^{7,8}$ Over-expression of Yap/TAZ leads to hepatocytes over proliferation and liver enlargement, while loss of Yap/TAZ in liver results in spontaneous liver necrosis as early as 4weeks of age in mice, which is probably due to loss of the transcriptional activity of Yap/ TAZ. ${ }^{910}$ More interestingly, the cytoplasmic retention of Yap/TAZ by phosphorylation and binding with 14-3-3 are involved in cell contact inhibition, over expressing of non-phosphorylatable Yap/TAZ leads to loss of contact inhibition and tissue over growth. ${ }^{7}$ It has also been shown that Yap/TAZ possesses the mechanosensing property: when cells are cultured on surfaces with great stiffness, Yap/TAZ is nuclear localized with great transcriptional activity; whereas when cells grow on elastic surface, Yap/TAZ is mostly cytoplasmic retained with less transcriptional activity. ${ }^{11}$

A more recent study led by the Piccolo group proposed that when cells grow with small attaching area, they experience low mechanical stresses, as they display decreased integrin-mediated focal adhesions, reduced actin stress fibers, and blunted cell contractility, which lead to Yap/TAZ inactivation. ${ }^{12}$ They found that YAP/TAZ serve as a link between tissue architecture and cell proliferation, and their inhibition also entails a remodeling of the F-actin cytoskeleton. ${ }^{12}$ Therefore there are two faces of Yap/TAZ's function during liver regeneration: on one hand, Yap/TAZ promote cell proliferation with its transcriptional activity right after the acute liver injury, on the other hand the phosphorylated YAP/TAZ act as a switch to stop cell proliferation 
by mediating cell contact inhibition and mechanosensing of the environment stiffness. Other than Hippo pathway, WNT and GPCR signaling pathways have also been recognized as important regulators of YAP/TAZ activity. ${ }^{13,14}$

\section{Signaling molecules involving mechanosensing in liver regeneration: 14-3-3}

14-3-3 is a binding partner of Yap/TAZ when they are phosphorylated and located in the cytoplasm. ${ }^{8}$ In mammalian cells, there are seven isoforms $(\beta, \varepsilon, \gamma, \eta, \sigma, \tau / \theta$ and $\zeta)$ of 14-3-3 and share highly conserved homology among all eukaryotic cells. Functioning to bind phophoserine/phosphothreonine targets, 14-33 proteins participate in phosphorylation-dependent protein-protein interactions. ${ }^{15}$ 14-3-3 proteins play crucial roles in regulating many cellular processes, including the maintenance of cell cycle checkpoints and DNA repair, apoptosis, cell differentiation and cytoskeleton dynamics. Recently, 14-3-3 protein was found to maintain normal cell cortical tension in Dictyostelium cells and mammalian cancer cells. ${ }^{16,17}$ When 14-3-3 protein is down regulated, cells exhibit significantly low surface tension and accompanied with cytokinesis failure. In a global proteomic survey study followed by Ingenuity Pathway Analysis, 14-3-3 was identified as the center of pathway networks at all the time points after $2 / 3$ hepatectomy under experimental conditions, suggesting a central role of this protein in regulating liver regeneration..$^{18}$ Upon mRNA expression pattern analysis of all 14-3-3 isoforms after $2 / 3$ partial hepatectomy in rat liver, up regulation of $14-3-3 \xi / \varepsilon$ and down regulation of $14-3-3 \sigma$ mRNA from 0 to $6 \mathrm{~h}$ were observed, which may play important roles in hepatocytes proliferation at the entry into S-phase. ${ }^{19}$

\section{Physical cues involving liver regeneration: hepatocyte swelling}

Hepatocyte swelling has been observed in many clinical scenarios from sepsis, hepatic resection, ischemia-reperfusion injury, and so on Pedersen $\mathrm{P},{ }^{20}$ Sadoshima J. ${ }^{21}$ Using various models of hepatocyte swelling, investigators have demonstrated changes in cell structure, such as membrane stretch, cytoskeleton remodeling, and alterations in cytoskeleton-membrane complexes. ${ }^{22}$ Other studies demonstrated a causal relationship between cell volume with the activation of many intracellular proliferative signals, such as MAPKs, PI3K and PKC pathways, NF- $\kappa \mathrm{B}$, STAT, and AP-1 transcription factors, and induction of immediate early genes of regeneration. ${ }^{22}$ Thus the physical changes of hepatocyte swelling were translated into chemical signals through mechanosensing molecules, possibly Yap/TAZ as discussed above.

\section{Summary}

Cells are susceptible to countless environmental factors, which includes both chemical and physical factors. In the past, numerous efforts have been focused on chemical cues on liver regeneration. This review is intended to draw more attention to physical cues on liver regeneration. An overall understanding of liver regeneration chemically and physically will not only lead to better understandings of other organ regeneration, it may also shed light on how cancer develops in various liver diseases.

\section{Acknowledgements}

None.

\section{Conflict of interest}

\section{References}

1. Fausto N, Campbell JS, Riehle KJ. Liver regeneration. Hepatology. 2006;43(2 Suppl 1):S45-S53.

2. Michalopoulos GK. Liver regeneration. $J$ Cell Physiol. 2007;213(2):286-300.

3. Riehle KJ, Dan YY, Campbell JS, et al. New concepts in liver regeneration. J Gastroenterol Hepatol. 2011;(26 Suppl 1):203-212.

4. Knight B, Yeoh GC, Husk KL, et al. Impaired preneoplastic changes and liver tumor formation in tumor necrosis factor receptor type 1 knockout mice. J Exp Med. 2000;192(12):1809-1818.

5. Duncan AW, Dorrell C, Grompe M. Stem cells and liver regeneration. Gastroenterology. 2009;137(2):466-481.

6. Erker L, Grompe M. Signaling networks in hepatic oval cell activation. Stem Cell Res. 2007;1(2):90-102.

7. Zhao B1, Wei X, Li W, et al. Inactivation of YAP oncoprotein by the Hippo pathway is involved in cell contact inhibition and tissue growth control. Genes Dev. 2007;21(21):2747-2761.

8. Schlegelmilch K, Mohseni M, Kirak O, et al. Yap1 acts downstream of alpha-catenin to control epidermal proliferation. Cell. 2011;144(5):782-795.

9. Dupont S, Morsut L, Aragona M, et al. Role of YAP/TAZ in mechanotransduction. Nature. 2011;474(7350):179-183.

10. Johnson R, Halder G. The two faces of Hippo: targeting the Hippo pathway for regenerative medicine and cancer treatment. Nat Rev Drug Discov. 2014;13(1):63-79.

11. Wu H, Xiao Y, Zhang S, et al. The Ets transcription factor GABP is a component of the hippo pathway essential for growth and antioxidant defense. Cell Rep. 2013;3(5):1663-1677.

12. Aragona M, Panciera T, Manfrin A, et al. A mechanical checkpoint controls multicellular growth through YAP/TAZ regulation by actinprocessing factors. Cell. 2013;154(5):1047-1059.

13. Yu FX, Zhao B, Panupinthu N, et al. Regulation of the Hippo-YAP pathway by G-protein-coupled receptor signaling. Cell. 2012;150(4):780-791.

14. Azzolin L, Zanconato F, Bresolin S, et al. Role of TAZ as mediator of Wnt signaling. Cell. 2012;151(7):1443-1456.

15. Wilker E, Yaffe MB. 14-3-3 Proteins--a focus on cancer and human disease. J Mol Cell Cardiol. 2004;37(3):633-642.

16. Zhou Q, Kee YS, Poirier CC, et al. 14-3-3 coordinates microtubules, Rac, and myosin II to control cell mechanics and cytokinesis. Curr Biol. 2010;20(21):1881-1889.

17. Zhou Q. 14-3-3 controls cell cortical tension in human cells. ASCB/IFCB Meeting Abstract 48. 2014.

18. Chen $\mathrm{XG}, \mathrm{Xu} \mathrm{CS}$. Proteomic analysis of the regenerating liver following 2/3 partial hepatectomy in rats. Biol Res. 2014;47(1):59.

19. Xue DM, Guo XQ, Chen R, et al. 14-3-3 gene expression in regenerating rat liver after $2 / 3$ partial hepatectomy. Genet Mol Res. 2015;14(1):2023-2030.

20. Pedersen P, Seeman T, Hasselgren PO. Protein synthesis and degradation in liver tissue following induction of septic peritonitis in rats. Acta Chir Scand. 1986;152:29-34.

21. Sadoshima J, Qiu Z, Morgan JP, et al. Tyrosine kinase activation is an immediate and essential step in hypotonic cell swelling-induced ERK activation and c-fos gene expression in cardiac myocytes. EMBO J. 1996;15(20):5535-5546.

22. Kim RD, Stein GS, Chari RS. Impact of cell swelling on proliferative signal transduction in the liver. J Cell Biochem. 2001;83(1):56-69.

Author declares that there is no conflict of interest. 